\title{
Miranda
}

Revue pluridisciplinaire du monde anglophone /

Multidisciplinary peer-reviewed journal on the English-

speaking world

19 | 2019

Rethinking Laughter in Contemporary Anglophone Theatre

\section{Édouard Marsoin, Melville et l'usage des plaisirs.}

\section{Mark Niemeyer}

\section{(2) OpenEdition}

\section{Journals}

\section{Édition électronique}

URL : http://journals.openedition.org/miranda/21457

DOI : $10.4000 /$ miranda. 21457

ISSN : 2108-6559

\section{Éditeur}

Université Toulouse - Jean Jaurès

\section{Édition imprimée}

Date de publication : 7 octobre 2019

\section{Référence électronique}

Mark Niemeyer, «Édouard Marsoin, Melville et l'usage des plaisirs. », Miranda [En ligne], 19 | 2019, mis en ligne le 14 octobre 2019, consulté le 16 février 2021. URL : http://journals.openedition.org/miranda/ 21457 ; DOI : https://doi.org/10.4000/miranda.21457

Ce document a été généré automatiquement le 16 février 2021.

\section{c) (i) $९$}

Miranda is licensed under a Creative Commons Attribution-NonCommercial-NoDerivatives 4.0 International License. 


\section{Édouard Marsoin, Melville et l'usage des plaisirs.}

Mark Niemeyer

\section{RÉFÉRENCE}

Édouard Marsoin, Melville et l'usage des plaisirs (Paris : Sorbonne Université Presses, 2019), 592 p., ISBN 979-10-231-0618-3.

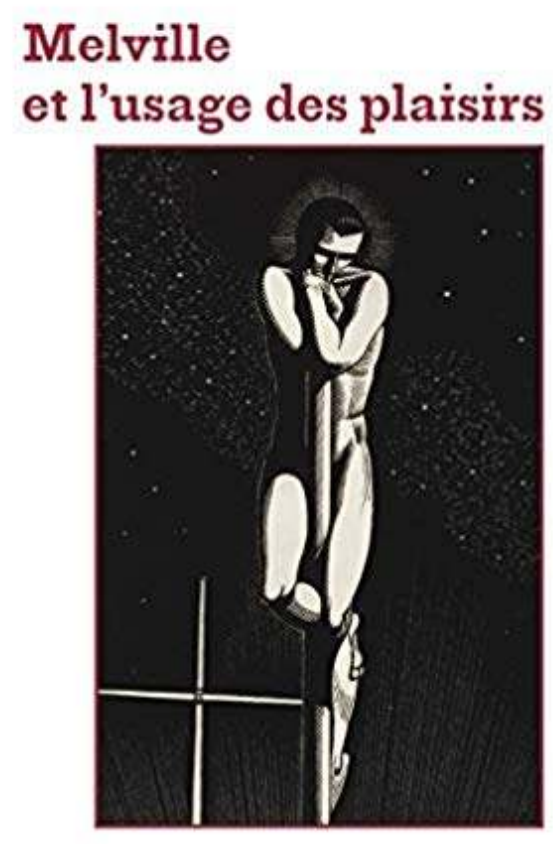

Édouard Marsoin 
1 Avec Melville et l'usage des plaisirs, Édouard Marsoin fait une contribution innovante et importante à la critique d'un des auteurs américains majeurs du dix-neuvième siècle. Innovante parce que le thème du plaisir n'est pas souvent associé à Herman Melville, dont la fiction, pour un grand nombre de lecteurs, comme le rappelle Marsoin, «serait 'noire', pessimiste et misanthrope » (12) - même s'il y a depuis longtemps des études qui montrent que cette image est réductrice. Et importante parce que cet ouvrage ambitieux de 592 pages analyse le discours melvillien lié au concept du plaisir de plusieurs points de vue, en utilisant des approches critiques variées (poétiques, esthétiques, psychanalytiques, économiques) dans la quasi-totalité de l'œuvre en prose de l'auteur (tous les romans et plusieurs nouvelles sont étudiés). Il s'agit, en fait, d'un nouveau regard sur Melville qui le montre dans une lumière plus joyeuse (dans certains cas), mais pas moins complexe ni moins ambigu. L'introduction explore les significations multiples de "plaisir» en l'associant au concept de la jouissance, en partie parce que chez Melville le plaisir et la souffrance sont souvent entremêlés. Le sujet du livre, en fait, est le plaisir dans un sens large, qui inclut, mais qui dépasse, bien entendu, le plaisir sexuel, comprenant « tous les types de plaisirs possibles, comme les plaisirs de la sociabilité, de la nourriture, de la boisson, du tabac, c'est-à-dire l'ensemble des plaisirs 'mondains', qui peuvent avoir une qualité sensuelle et érotique sans être néanmoins sexuels » (27). Et c'est tout à fait naturel si Édouard Marsoin fait appel à Montaigne, philosophe très apprécié par Melville, quand il aborde plus spécifiquement l'idée de «l'usage » des plaisirs parce qu'à l'instar de Montaigne il constate que «Les plaisirs mondains, sensuels sans être sexuels » sont "toujours corporels, leur 'usage' toujours un certain usage des corps " (28). Il faut noter également que si Melville et l'usage des plaisirs est surtout une étude d'un certain discours littéraire sur le plaisir, le contexte biographique et historique d'une œuvre clairement ancrée dans le dixneuvième siècle américain n'est pas oublié.

2 L'ouvrage est divisé en quatre parties comprenant douze chapitres. «Poétique des plaisirs ", la première partie, montre le lien étroit entre les références aux plaisirs dans le texte melvillien et une certaine conception de la littérature. Comme l'écrit Édouard Marsoin, pour cet auteur qui aimait lui-même manger, boire, fumer et discuter avec des amis - surtout avec des amis littéraires (on pense notamment bien entendu à Nathaniel Hawthorne), « plaisirs et discours, mots et matières, sont inséparables » (39). Assez naturellement donc, « Nourriture, boissons et tabac sont des éléments centraux de la poétique melvillienne, car ils y définissent la texture du réel et du symbolique » (41). Pour Marsoin, «Les images et métaphores des plaisirs (en particulier culinaires) sont en effet, en raison de leur récurrence, plus que de simples images, mais aussi des structures signifiantes cohérentes qui combinent fonctions référentielles et fonctions sémiotiques pour construire des mondes fictifs originaux » (43). Ce que Marsoin appelle un « monde-table » est omniprésent dans les écrits de Melville, notamment dans Mardi, son troisième livre, publié en 1849 , où, par exemple, « l'aigre-doux constitue un schème récurrent qui s'intègre à la dialectique des plaisirs et de la mort qui informe le roman » (60). Pierre, publié en 1852, est également « une romance de l'aigre-doux, où le sucre se révèle amer, et où l'amertume devient un choix de régime assumé » (63). Dans Billy Budd, œuvre posthume, pour prendre un dernier exemple, un « effet sucrant » «fait partie des représentations genrées qui féminisent Billy tout en lui donnant un effet de domestication sur l'équipage » (65). "La Gourmandise des corps dans Typee» est le deuxième chapitre de la première partie, le seul consacré exclusivement à un seul livre de Melville. Ici, Marsoin souligne avec raison la tendance chez Tommo, le narrateur du 
premier livre de l'auteur, à regarder tous les plaisirs de l'île paradisiaque où il se trouve avec suspicion: "pour lui, ces plaisirs ne peuvent être gratuits, ils doivent cacher quelque chose (probablement une douleur, puisque pour l'Occident tout plaisir est la récompense d'une douleur)» (91). Il y a, en effet, dans Typee, «quelque chose comme une résistance au plaisir qui est une expérience des limites du jouir » (92). Le thème du cannibalisme est notamment exploré dans ce contexte. Et même si ce sujet a déjà été étudié chez Melville, Marsoin ajoute une perspective originale en le plaçant clairement dans le contexte du plaisir. Il souligne, par exemple, que c'est bien Tommo qui semble être obsédé par l'idée que les Taïpis puissent être des cannibales et c'est donc lui qui casse l'image d'un paradis sur Terre. Plus profondément, Marsoin explore le lien entre le caché et le plaisir. Comme il l'explique, par exemple, "Lorsque Tommo donne une robe à Faïaoahé, d'ordinaire dénudée, sa cheville n'en est que plus érotique ('la plus ravissante cheville du monde') » (105). Cette observation est sans doute juste, mais l'on pourrait noter également que Melville s'amuse ici (encore une autre forme de plaisir) en faisant cette remarque qui non seulement se moque d'un certain puritanisme qui serait choqué par cette cheville exposée au monde mais qui offre également un clin d'œil taquin à Washington Irving qui note dans « The Legend of Sleepy Hollow » que Katrina Van Tassel, la jeune coquette du village, portait un «jupon court le plus provoquant, afin de mettre en évidence le plus joli pied et cheville qui fussent dans le pays d'alentour». Le chapitre sur «Plaisirs et discours: les banquets melvilliens» continue l'étude des plaisirs mondains. Marsoin note, par exemple, que dans "The Paradise of Bachelors and the Tartarus of Maids " Melville met en scène "les plaisirs coupables des célibataires" (111). "Coupables» ou non, il est clair que les plaisirs décrits dans les œuvres de Melville sont souvent partagés par des hommes éloignés du monde des femmes, ce qui n'est peut-être pas étonnant chez un auteur qui a commencé sa vie de jeune marié en s'établissant dans une maison avec son épouse, sa mère, ses quatre sœurs célibataires ainsi qu'avec son frère Allan et l'épouse de ce dernier. Comme Marsoin le note, dans Mardi « on dénombre au moins neuf scènes de banquet, qui se déclinent en petits déjeuners, déjeuners et dîners " (119), créant un festin, ici encore, masculin, qui semble sans fin. Mais la fête n'est pas sans ambivalence. Le narrateur remarque, en fait, en parlant d'un des repas grandioses de Mardi, que « comme dans la plupart des banquets publics, les convives sont très nombreux et beaucoup quittent cette table débordante avec le ventre creux» (119). Et les mots sont aussi les mets. Marsoin souligne que «Comme les autres banquets melvilliens, le banquet mardien est donc un espace intertextuel, c'est-à-dire un dispositif d'ingestion, digestion et production de discours » (124).

3 La deuxième partie, "Sémiotique, épistémologie et esthétique des plaisirs » se focalise sur la quête de la vérité dans la fiction de Melville. Cette quête est liée au plaisir dans les domaines de la sémiotique, de l'épistémologie et de l'esthétique parce que, comme l'affirme Édouard Marsoin, chez Melville « il ne peut y avoir de vérité que dans un corps et dans une âme. [...] c'est toujours un corps qui pense qui recherche la vérité » (141). Les signes et leur interprétation sont centraux dans l'œuvre melvillienne (on pense, par exemple, au chapitre «Le Doublon» dans Moby-Dick). Et comme Marsoin l'explique, « face à l'alphabet du monde, l'impulsion sémiotique offre deux modalités affectives de déchiffrement : entamer un processus douloureux et infini, comme Pierre qui, comme Achab, fait le choix de la souffrance, ou au contraire suivre son bon plaisir et faire le choix du plaisir, comme Israël dans Israel Potter » (145). Mais si le chapitre « Melville et les signes» explore intelligemment la sémiotique melvillienne, notamment dans 
«Benito Cereno » (1855) et Moby-Dick (1851), on s'éloigne parfois du sujet du plaisir. Dans le chapitre 5, "L'Épistémè de la jouissance », on y revient plus clairement, mais parfois plus par l'absence du plaisir que par sa présence. Comme Marsoin le note très justement, Pierre, pour sa part, confond souffrance et vérité : «Cette erreur constitue le cœur du roman: Pierre s'aventure dans les profondeurs de son âme au prix de douleurs immenses et fait de la souffrance le signe de la vérité » (161). Dans Moby-Dick, Ismaël, par contre, trouve du plaisir dans sa quête de la vérité, même si elle ne peut pas aboutir: "Ce qui motive le discours ismaélien», écrit Marsoin, "n'est pas véritablement une volonté de savoir, mais plutôt une volonté de jouer et jouir de l'impossibilité de ce savoir» (167). Enfin, «Jouir d'un objet, nous montre Ismaël, c'est jouir du cheminement vers cet objet, plus que de son atteinte. Le mouvement simultané de construction et déconstruction est au cœur de la jouissance du texte dans MobyDick» (177). Le lien entre l'humour et la quête de la vérité est également exploré dans ce chapitre. L'humour, en fait, est une autre façon chez Melville de rapprocher le monde des connaissances et le monde du plaisir. «Physiologie et esthétique de la vérité ", le dernier chapitre dans cette partie, se penche sur la question de l'importance relative du plaisir et de la vérité dans la fiction de Melville, écrivain de la période romantique qui n'a jamais embrassé complètement le culte du plaisir que l'on retrouve chez certains auteurs, notamment britanniques, de cette période. Mais pour Marsoin, «Si Melville n'abandonne jamais tout à fait l'objet de la vérité au profit du plaisir, il ne manque pas d'interroger ce qui fait la vérité du plaisir esthétique » (233).

"Éthique et diététique des plaisirs ", la troisième partie de Melville et l'usage des plaisirs, traite de la question des "mises en forme éthique par lesquelles des sujets fictifs règlent leurs rapports à la vie et au plaisir, et élaborent ainsi des régimes » (257). Marsoin souligne l'importance chez Melville du contraste entre le plaisir et la douleur en déterminant « une posture éthique tenable face à l'instabilité du monde » (259). La notion d'antipéristase est au centre de son analyse dans cette partie, notion d'origine antique et renaissante qui désigne une opposition entre deux qualités contraires qui rend chacune plus forte par comparaison. Comme l'écrit Marsoin, «L'expérience de la vie est chez Melville similaire à celle du plaisir, dont la compréhension ne relève pas simplement de la notation d'une sensation, ou de la description d'un état, mais d'une élaboration formelle qui définit le plaisir (et la vie) par contraste avec la souffrance (et la mort)» (262). Ce principe antipéristatique, d'après Marsoin, "traverse toute la fiction melvillienne " (263). On peut en voir un exemple simple vers le début de MobyDick quand Ismaël partage un lit avec Quiqueg et fait la remarque que l'on ne peut pas complètement apprécier le bonheur de la chaleur sous les couvertures si l'on n'a pas au moins une petite partie du corps exposée en même temps à la fraîcheur de la chambre (263). Mais le principe va évidemment beaucoup plus loin. Dans Mardi, par exemple, "L'échappée du narrateur et de Jarl est dès le début marquée par le contraste de la joie et du danger : 'Ah ! malgré notre terrible situation, avec quelle joie nous voguions !' » (271). Pour Marsoin, « la composante la plus centrale du tragique melvillien, ce n'est pas la souffrance (la souffrance est bête), mais plutôt la possibilité du plaisir et de la joie, l'expérience antipéristatique d'un monde composite, où terreur et joie, souffrances et plaisirs, se nourrissent mutuellement» (288). Le dernier chapitre de cette partie, "Régimes et régimes de soi: les quatre ascètes" regroupe de façon intéressante et innovante quatre personnages de l'œuvre melvillienne - Achab, Pierre, Bartleby et Franklin - qui sont tous « des contempteurs de corps qui prêchent la séparation du corps et de l'âme » (328). Ici également, il s'agit surtout de l'absence de 
plaisir, mais c'est une absence qui peut apporter néanmoins une autre sorte de plaisir. Par exemple, "Ce que recherche Achab dans son régime ascétique », écrit Marsoin, "c'est le contrôle de soi et des autres" (337). Mais comme il le note aussi, «La délectation d'Achab dans le chapitre 'The Quarter-Deck', où 'peu de temps après l'épisode de la pipe', il met en place son pacte diabolique avec l'équipage par la distribution d'alcool, est le meilleur exemple d'une jubilation qui naît dans le goût du contrôle » (341).

5 La quatrième et dernière partie s'appelle "Socialité, politique et économie des plaisirs » et se concentre sur le contexte social, dans un sens large, du plaisir. Comme l'explique Marsoin, «le plaisir, dans la fiction de Melville, n'est pas une simple affaire de physiologie individuelle, mais aussi l'effet de mécanismes collectifs qui déterminent ses conditions de possibilité et d'expérimentation » (394). Le premier chapitre de cette partie analyse l'amitié, une valeur pour Melville, comme l'indique Marsoin, « centrale dans sa fiction, comme dans sa vie» (397). Il y a, bien entendu, un certain homoérotisme dans plusieurs de ces amitiés, notamment dans celle entre Ismaël et Quiqueg, mais chez Melville rien n'est jamais uniquement physique. Comme l'écrit Marsoin, "on pourrait dire que l'éros et philia grecs - tous deux principes de cheminement vers le Beau et le Bien chez Platon - trouvent dans la rencontre de Quiqueg et Ismaël une lecture où l'attrait physique est inséparable de son pendant spirituel, la vertu» (432). Dans «Benito Cereno» la question d'amitié devient plus complexe et plus ambiguë avec Amasa Delano, le capitaine américain naïf, perdu devant un jeu de masques de l'amitié où il ne peut jamais être certain de qui a des intentions amicales. Mais c'est dans Billy Budd où l'amitié se transforme en haine. Dans ce roman, presque achevé à la mort de Melville en 1891, comme Marsoin le note, «les rapports entre Billy et ses bourreaux semblent a priori amicaux» (454). Mais Claggart et le capitaine Vere ne sont pas des amis de Billy. Le problème, d'après Marsoin, qui suit les thèses de Eve K. Sedgwick et Barbara Johnson, est qu'à la fin du dix-neuvième siècle ce n'était plus possible pour Melville d'offrir un portrait d'une amitié homoérotique comme innocent et réalisable, même dans une œuvre de fiction, parce que ce moment historique coïncide avec l'émergence d'une identité homosexuel moderne que la société se sentait obligée de rejeter (464). Billy, qui paraît sortir d'un temps peut-être plus innocent, est, comme Marsoin l'écrit, « un archaïsme, une espèce en danger : il meurt de son anachronisme » (469). Le chapitre 11 se focalise sur Redburn et White-Jacket et analyse le monde des navires comme un champ où les plaisirs sont réglementés jusqu'au dernier détail et servent de soupape de décompression, à l'instar, comme l'indique Marsoin, des journées de liberté permises aux esclaves américains entre Noël et le Jour de l'an (484). Le livre se termine avec un chapitre sur « Économie(s) du plaisir et de la douleur » qui explore la question de travail et l'influence de la "civilisation » américaine et européenne sur les Polynésiens, notamment, bien entendu, dans Typee et Omoo. Sans surprise, les plaisirs polynésiens souffrent des efforts de cette mission civilisatrice.

$6 \quad$ Melville et l'usage des plaisirs est un tour de force impressionnant qui offre une analyse du discours melvillien lié au plaisir qui est profonde, intelligente et éclairante. Marsoin montre dans cette étude non seulement une très bonne connaissance de Melville, mais également une grande familiarité avec toute la littérature américaine du dix-neuvième siècle. Ses analyses fines font appel à des approches critiques variées, sans jamais être déformées par une trop grande adhésion aux idées de tel ou tel théoricien invoqué. Ce grand livre, publié dans un format agréable par Sorbonne Université Presse, est un 
remaniement d'une thèse soutenue en 2016 sous la direction de Philippe Jaworski, le doyen des études melvilliennes en France. Si le résultat offre un traitement clair et innovant de son sujet, dans un style soigné (même si parfois un peu dense), il faut dire qu'avec sa structure autour de quatre grands thèmes, qui est tout à fait logique et cohérente, en combinaison avec son traitement de toute l'œuvre de fiction de Melville, ce livre est de plutôt destiné aux lecteurs qui connaissent déjà bien leur Melville (il y a souvent des exemples tirés de trois ou quatre différents romans ou nouvelles sur une seule page). En tout cas, Melville et l'usage des plaisirs fera date dans les études melvilliennes. Une belle étude sur le plaisir chez Herman Melville. Quel beau cadeau pour cet auteur et pour ceux qui l'apprécient en cette année 2019 qui marque le $200^{\text {ème }}$ anniversaire de ce grand auteur américain.

\section{INDEX}

Mots-clés : jouissance, Melville, plaisir

Keywords : enjoyment, jouissance, Melville, pleasure

\section{AUTEURS}

\section{MARK NIEMEYER}

Professeur

Université de Bourgogne

Mark.Niemeyer@u-bourgogne.fr 